Research Article

\title{
Effect of Lifestyle Factors on Hypertension by Constitution Type: A Large Community-Based Study
}

\author{
Ji-Eun Park $\left(\mathbb{D},{ }^{1}\right.$ Chol Shin $\mathbb{D D}^{2}$ and Siwoo Lee $\mathbb{D}^{1}$ \\ ${ }^{1}$ Future Medicine Division, Korea Institute of Oriental Medicine, Daejeon, Republic of Korea \\ ${ }^{2}$ Institute of Human Genomic Study, College of Medicine, Korea University Ansan Hospital, Ansan, Republic of Korea \\ Correspondence should be addressed to Siwoo Lee; bfree@kiom.re.kr
}

Received 28 August 2019; Accepted 13 November 2019; Published 4 December 2019

Academic Editor: Christopher Worsnop

Copyright (c) 2019 Ji-Eun Park et al. This is an open access article distributed under the Creative Commons Attribution License, which permits unrestricted use, distribution, and reproduction in any medium, provided the original work is properly cited.

Background. The risk of hypertension differs according to lifestyle factors and individual constitution types. The aim of this study was to investigate the effect of lifestyle factors on hypertension and to assess whether those effects differ according to the constitution types. Methods. A total of 5,793 men and women were recruited between 2012 and 2014. Odds ratios for hypertension associated with constitution types and lifestyle factors were estimated. Lifestyle factors included smoking status, body mass index, alcohol consumption, physical activity, and sleep quality. Constitution types were estimated based on the Sasang constitutional medicine as the TE type, SE type, and SY type. Results. The risk of hypertension was significantly higher for SY (odds ratio 1.25 (95\% confidence interval 1.03 to 1.52) and TE types (1.38 (1.10 to 1.74)) than the SE type even with adjustment of health behaviors. Compared with individuals who had an unhealthy lifestyle, those with healthy lifestyle scores showed significantly lower risk of hypertension in only SY (odds ratio 0.62 (95\% confidence interval 0.48 to 0.81$)$ ) and TE types $(0.69$ ( 0.58 to 0.81$)$ ). The difference in risk for hypertension among constitution types was decreased with a healthy lifestyle (1.34 in SY and 2.35 in TE types, as compared with the SE type) versus an unhealthy lifestyle (2.21 in SY and 3.64 in TE types, as compared with the SE type). Conclusion. The risk of hypertension was different by Sasang constitution types. The impact of lifestyle factors differed according to Sasang constitution types, and the difference in risk of hypertension among constitution types was decreased with a healthy lifestyle.

\section{Introduction}

Hypertension is a major disease leading to various secondary diseases, including myocardial infarction, stroke, and renal failure [1]. In 2010, over $30 \%$ of the world's adults had hypertension [2], and the estimated annual death rate associated with hypertension is increasing [3]. In the Republic of Korea, the prevalence of hypertension showed among adults in 2016 was nearly $30 \%$ [4], accounting for the greatest proportion of healthcare expenditures [5].

As reported in previous studies, lifestyle factors are known to be related to the risk for hypertension [6], and a healthy lifestyle could reduce the incidence of hypertension. Shafieyan et al. showed that the score of healthpromoting lifestyle profile in hypertension participants was significantly lower than the score in healthy participants without hypertension [7]. Another study investigating the risk of developing hypertension reported that participants with healthy lifestyle exhibited $46 \%$ relative reduction in the risk of developing hypertension compared with participants with unhealthy lifestyle [8].

However, the effect of lifestyle factors might differ according to individual factors such as race, age, and sex. One study showed that people from South Asia need more physical activity to obtain the same cardiometabolic levels as white Europeans [9]. In another study, healthy lifestyle showed weaker association with hypertension in older compared with younger age [10]. The results of these studies showed that the effect of healthy lifestyle could be different by individual's characteristics.

As one of the medicines considering individual's physiologic and physical characteristics, Sasang constitutional 
medicine classifies people into four constitutions based on their traits: Tae-Yang (TY), Tae-Eum (TE), So-Yang (SY), and So-Eum (SE) types. The traits of each constitution include psychological characteristics such as body structure, function, and metabolism. Each Sasang constitution has different strong and weak organs. For example, individuals with the TE constitution have a strong lung and a weak liver [11]. Because of these differences between constitutions, some medicines or herbs might cause adverse effects in individuals with certain constitutions, despite being effective in treating those with other constitutions [12].

As each constitution has different physiological features (such as the congenital strength/weakness of the internal organs), the susceptibility to disease also differs between constitutions [13]. For example, the SY type has weak kidney function and is vulnerable to renal disease while the SE type has weak spleen function and frequently has digestive malfunctions [12]. Previous studies have showed that the risk of diseases including hypertension [14, 15], prehypertension [16], metabolic syndrome [13, 17], diabetes [18], abdominal obesity [19], and cancer [20] differ according to Sasang constitution types.

Because of the differing disease susceptibility of each constitution type, the effect of risk factors on chronic diseases such as hypertension might also differ according to constitution. Previous studies have showed that predictors for various diseases such as metabolic syndrome [21] and nonalcoholic fatty liver disease [22] were different according to Sasang constitution types. For example, higher body mass index (BMI) is a significant risk factor of nonalcoholic fatty liver disease in the SY type, but it is not significant in the TE type [22].

Although several studies have assessed the risk of hypertension by constitution types, there are no studies investigating the effect of lifestyle on hypertension by different constitution types. In this study, we aimed to investigate the effect of lifestyle factors on hypertension and to assess whether those effects differ by Sasang constitution types.

\section{Methods}

2.1. Study Population. All data, including Sasang constitution types and lifestyle factors, were compiled from the Korean Medicine Data Center (KDC) of the Korea Institute of Oriental Medicine (KIOM), which is the largest clinical database of Sasang constitution and Korean medicine in Korea [23]. KIOM collected these data from June 2012 to July 2014 as a part of community-based cohort study, which was conducted for the general population aged 40-69 years living in urban and rural areas (Ansan, Ansung) in Korea [24]. All participants underwent a comprehensive health examination and on-site interview to collect information on disease status, lifestyle, and Sasang constitution types. Informed consent was obtained from all participants when data were collected. The Institutional Review Board at the KIOM reviewed and approved this study (I-1809/006/001).
2.2. Classification of Sasang Constitution Types. To assess Sasang constitution types, the Korean Sasang constitutional diagnostic questionnaire (KS-15) was used. The KS-15 was developed by the KIOM based on information on the body shape, temperament, and symptoms, and validated [25]. This questionnaire consists of 15 items including current height/ weight, six personality questions (e.g., broad-minded? extraverted?) and eight symptom questions related to physiological functions (e.g., digest well? dislike cold or heal?). Detailed information on KS-15 was reported in the previous study [25].

2.3. Assessment of Lifestyle Factors. Lifestyle factors for hypertension were selected based on previous studies, included smoking, alcohol consumption, obesity, physical activity, and sleep quality [26-28]. Smoking was defined as current smoker, former smoker, and never smoker. Obesity was assessed using BMI and categorized as normal weight $\left(<23 \mathrm{~kg} / \mathrm{m}^{2}\right)$, overweight $\left(\geq 23 \mathrm{~kg} / \mathrm{m}^{2}\right)$, and obese $(\geq 25 \mathrm{~kg} /$ $\mathrm{m}^{2}$ ) based on the World Health Organization Western Pacific Region criteria [29]. Alcohol intake was measured in $\mathrm{g} /$ day and participants were categorized as nondrinker $(<5 \mathrm{~g} /$ day), moderate drinker ( $<15 \mathrm{~g} /$ day), and heavy drinker ( $\geq 15 \mathrm{~g} /$ day). Frequency, duration, and type of physical activity were measured and defined in total metabolic equivalents (MET) minutes per week. For the analysis, $\geq 500$ METminutes/week was used as the criterion for healthy physical activity levels [30]. Sleep quality was measured using the Pittsburgh Sleep Quality Index. This questionnaire measures seven components including subjective sleep quality, sleep latency, sleep duration, habitual sleep efficiency, sleep disturbances, use of sleeping medications, and daytime dysfunction over the previous month. A total score of $\leq 5$ is considered to indicate good sleep quality [31].

To determine the scores for a healthy lifestyle, each factor was given a score of 1 if the criterion for low risk was satisfied, i.e., never smoker, BMI $<23 \mathrm{~kg} / \mathrm{m}^{2}$, alcohol consumption $<5 \mathrm{~g} /$ day, physical activity $\geq 500$ METminutes/ week, and good sleep quality. Even though Sotos-Prieto et al. used the lifestyle score considering different weights of each lifestyle [32], the lifestyle score measurement reflecting the impact of each lifestyle is not clear until now. So, in this study, each factor of lifestyle scored one equally based on previous studies $[28,33,34]$. The total lifestyle score was the sum of these five scores, ranging from 0 to 5, with a higher score indicating a healthier lifestyle. All individuals were classified into either the unhealthy (score 0-2) and healthy lifestyle (score 3-5) groups, based on their total lifestyle score.

2.4. Diagnosis of Hypertension. Hypertension was identified through blood pressure assessment and the standardized questionnaire asking whether the participants get treatments for hypertension. If they had an SBP $\geq 140 \mathrm{mmHg}$ or DBP $\geq 90 \mathrm{mmHg}$, or reported the use of antihypertensive medication, they were considered to have hypertension. 
2.5. Statistical Analysis. Each lifestyle factor and hypertension prevalence were summarized using descriptive analysis and expressed as mean \pm standard deviation for the total lifestyle score, which is continuous variable and frequencies or percentages for categorical variables. The differences in lifestyle factors and hypertension prevalence were evaluated using the chi-square test. To estimate the odds ratio (OR) of hypertension prevalence associated with constitution types and lifestyle factors, we conducted a univariate or multivariate logistic regression analysis. Age and sex were included in the logistic analysis as covariates. The risk of hypertension according to constitution types was also calculated and displayed. Statistical analysis was performed with $R$ version 3.3.1 (the $R$ Project for Statistical Computing, Vienna, Austria), and $p$ values less than 0.05 were considered statistically significant.

\section{Results}

3.1. Prevalence of Lifestyle Factors and Hypertension by Constitution. A total of 5,796 individuals participated in this study. One participant with no information for Sasang constitution types and two participants with missing sleeping data were excluded from the analysis. After these exclusions, we analyzed data of 5,793 participants, comprising $57.3 \%$ of the TE type, $25.4 \%$ of the SY type, and $17.3 \%$ of the SE type. There were no individuals with the TY type among participants. The prevalence of lifestyle factors was significantly different among Sasang constitution types ( $p<0.001$ for all lifestyle factors). The proportion of never smokers was highest for the SE type, with $69.6 \%$. Obesity showed the highest proportion for the TE type, with $69 \%$; there were no obese individuals of the SE type. In addition, the SE type showed the highest proportion of nondrinkers (77.6\%) compared with $66.5 \%$ for TE and $68.4 \%$ for SY types. Individuals with more than 500 METminutes/week of physical activity were most commonly of the SY type (55.4\%), compared with $48.7 \%$ of TE and $47.3 \%$ of SE type.

A total of $16.5 \%$ individuals of the SE type had all five healthy lifestyle factors, compared with only $0.6 \%$ of the TE type $(p<0.001)$. The mean number of healthy lifestyle factors was also significantly different among constitution types, with 2.54 for TE, 3.05 for SY, and 3.50 for SE types $(p<0.001)$. Significant differences were noted in hypertension prevalence among the three Sasang constitution types. The hypertension prevalence was highest for the TE type, with $53.5 \%$; this prevalence was $39.8 \%$ in SY and $30.8 \%$ in SE types $(p<0.001)$ (Table 1$)$.

3.2. Risk of Sasang Constitution for Hypertension. Compared with the SE type, SY and TE types showed significantly higher risk for hypertension. The OR for hypertension prevalence was 1.51 (95\% confidence interval (CI): $1.27,1.80)$ in SY and 2.76 (95\% CI: 2.37, 3.24) in TE types. This difference was still significant after adjusted sex, age, and health behaviors using multivariate logistic regression analysis, with 1.25 (95\% CI: 1.03, 1.52) in SY and 1.38 (95\% CI: $1.10,1.74)$ in TE compared with SE (Table 2).

3.3. Risk of Lifestyle Factors for Hypertension. In univariate analysis, ORs of former smokers (OR: 1.17, 95\% CI: 0.97, 1.40 ) and never smokers (OR: $0.98,95 \%$ CI: $0.79,1.21$ ) were not significantly different compared with current smokers. Compared with normal BMI, ORs for hypertension were significantly higher among overweight (OR 1.60, 95\% CI: $1.39,1.86)$ and obese individuals (OR 3.06, 95\% CI: 2.68, 3.50). Compared with nondrinkers $(<5 \mathrm{~g} /$ day), the risk of hypertension was not significant in moderate drinkers $(\geq 5$ to $<15 \mathrm{~g} /$ day), with OR 1.14 (95\% CI: 0.94, 1.37); however, this risk was significantly higher in heavy drinkers $(\geq 15 \mathrm{~g} /$ day) than nondrinkers: OR 1.52 (95\% CI: 1.29, 1.79). The effect of physical activity was not significantly different among the groups. Individuals with good sleep quality showed significantly lower risk of hypertension: OR 0.90 (95\% CI: 0.70, 0.90). Compared with the unhealthy lifestyle group (total lifestyle score $0-2$ ), the healthy lifestyle group (total score 3-5) had significantly lower risk for hypertension with OR 0.57 (95\% CI: 0.50, 0.65). In the multivariate model including lifestyle factors and constitution type, all factors except smoking and sleep quality were significant (Table 2).

3.4. Risk of Lifestyle Factors for Hypertension by Constitution. The effect of lifestyle factors differed according to Sasang constitution types. No lifestyle factors were significant for the SE type although overweight was marginally significant. For the SY type, both overweight (OR 1.38, 95\% CI: 1.09, 1.74 ) and obese (OR 1.53, 95\% CI: 1.00, 2.33) groups showed a higher risk of hypertension, compared with the normal BMI group. The risk of hypertension was marginally significant for heavy drinkers, and effect of smoking, physical activity, and sleep quality was not significant for the SY type.

For the TE type, overweight (OR 1.47, 95\% CI: 1.03, 2.11 )/obesity (OR 2.85, 95\% CI: 2.02, 4.04), heavy drinking (OR 1.47, 95\% CI: 1.18, 1.84), and physical activity (OR 0.75 , 95\% CI: 0.61, 0.92 in <500 MET minutes/week; OR 0.75, 95\% CI: $0.66,0.93$ in $\geq 500$ METminutes/week) had significant impacts on the risk of hypertension. Good sleep quality showed marginal significance, with OR 0.86 (95\% CI: 0.73, 1.01) (Table 3).

3.5. Risk for Hypertension by Constitution and Lifestyle. Individuals of the SE type with a healthy lifestyle did not show a significantly different risk of hypertension, in comparison with those of the SE type who had an unhealthy lifestyle. SY type with a healthy lifestyle showed a significantly lower hypertension risk than the SY type with an unhealthy lifestyle: OR 0.62 (95\% CI: $0.48,0.81)$. TE type with a healthy lifestyle had significantly lower risk of hypertension than the TE type with an unhealthy lifestyle (OR 0.69, 95\% CI: $0.58,0.81$ ) (Table 4(A)). 
TABle 1: Prevalence of lifestyle factors and hypertension by Sasang constitution types.

\begin{tabular}{|c|c|c|c|c|c|c|}
\hline & & So-Eum & So-Yang & Tae-Eum & Total & $p$ value \\
\hline \multirow{3}{*}{ Age } & $<55$ & $219(21.6)$ & $335(22.8)$ & $718(21.6)$ & $1,272(22.0)$ & \multirow{3}{*}{0.7773} \\
\hline & $\geq 55,<65$ & $413(41.1)$ & $580(39.5)$ & $1,375(41.4)$ & $2,368(40.9)$ & \\
\hline & $\geq 65$ & $373(37.1)$ & $554(37.7)$ & $1,226(36.9)$ & $2,153(37.2)$ & \\
\hline \multirow{2}{*}{ Sex } & Male & $390(38.8)$ & $713(48.5)$ & $1,587(47.8)$ & $2,690(46.4)$ & \multirow{2}{*}{$<0.001$} \\
\hline & Female & $615(61.2)$ & $756(51.5)$ & $1,732(52.2)$ & $3,103(53.6)$ & \\
\hline \multirow{3}{*}{ Smoker } & Current & $154(15.3)$ & $207(14.1)$ & $390(11.8)$ & $751(13.0)$ & \multirow{3}{*}{$<0.001$} \\
\hline & Former & $152(15.1)$ & $371(25.3)$ & $848(25.5)$ & $1,371(23.7)$ & \\
\hline & Never & $699(69.6)$ & $891(60.7)$ & $2,081(62.7)$ & $3,671(63.4)$ & \\
\hline \multirow{3}{*}{ BMI (kg/m2) } & $<23$ & $936(93.1)$ & $741(50.4)$ & $172(5.2)$ & 1,849 (31.9) & \multirow{3}{*}{$<0.001$} \\
\hline & $\geq 23,<25$ & $69(6.9)$ & $613(41.7)$ & $855(25.8)$ & $1,537(26.5)$ & \\
\hline & $\geq 25$ & $0(0.0)$ & $115(7.8)$ & $2,292(69.0)$ & 2,407 (41.6) & \\
\hline \multirow{3}{*}{ Alcohol consumption (g/day) } & $<5$ & $780(77.6)$ & $1,005(68.4)$ & $2,206(66.5)$ & $3,991(68.9)$ & \multirow{3}{*}{$<0.001$} \\
\hline & $\geq 5,<15$ & $83(8.3)$ & $160(10.9)$ & $408(12.3)$ & $651(11.2)$ & \\
\hline & $\geq 15$ & $142(14.1)$ & $304(20.7)$ & $705(21.2)$ & $1,151(19.9)$ & \\
\hline \multirow{3}{*}{ Physical activity (METminutes/week) } & 0 & $321(31.9)$ & $394(26.8)$ & $1,016(30.6)$ & $1,731(29.9)$ & \multirow{3}{*}{$<0.001$} \\
\hline & $>0,<500$ & $209(20.8)$ & $261(17.8)$ & $688(20.7)$ & $1,158(20.0)$ & \\
\hline & $\geq 500$ & $475(47.3)$ & $814(55.4)$ & $1,615(48.7)$ & $2,904(50.1)$ & \\
\hline \multirow{7}{*}{ Total lifestyle score } & 0 & $2(0.2)$ & $18(1.2)$ & $95(2.9)$ & $115(2.0)$ & \multirow{7}{*}{$<0.001$} \\
\hline & 1 & $31(3.1)$ & $135(9.2)$ & $441(13.3)$ & $607(10.5)$ & \\
\hline & 2 & $134(13.3)$ & $314(21.4)$ & $978(29.5)$ & $1,426(24.6)$ & \\
\hline & 3 & $298(29.7)$ & $464(31.4)$ & $1,212(36.5)$ & $1,971(34.0)$ & \\
\hline & 4 & $374(37.2)$ & $375(25.5)$ & $573(17.3)$ & $1,322(22.8)$ & \\
\hline & 5 & $166(16.5)$ & $166(11.3)$ & $20(0.6)$ & $352(6.1)$ & \\
\hline & Mean \pm SD & $3.50 \pm 1.03$ & $3.05 \pm 1.18$ & $2.54 \pm 1.03$ & $2.83 \pm 1.13$ & \\
\hline \multirow{2}{*}{ Lifestyle } & Unhealthy & $167(16.6)$ & $467(31.8)$ & $1,514(45.6)$ & $2,148(37.1)$ & \multirow[b]{2}{*}{$<0.001$} \\
\hline & Healthy & $838(83.4)$ & $1,002(68.2)$ & $1,805(54.4)$ & $3,645(62.9)$ & \\
\hline \multirow[b]{2}{*}{ Hypertension } & No & $695(69.2)$ & $885(60.2)$ & $1,544(46.5)$ & $3,124(53.9)$ & \multirow[b]{2}{*}{$<0.001$} \\
\hline & Yes & $310(30.8)$ & $584(39.8)$ & $1,775(53.5)$ & $2,669(46.1)$ & \\
\hline Total & & $1,005(17.3)$ & $1,469(25.4)$ & $3,319(57.3)$ & $5,793(100)$ & \\
\hline
\end{tabular}

BMI: body mass index, SD: standard deviation, and MET: metabolic equivalents. Data presented as $n$ (\%).

TABLE 2: Odds ratios of hypertension for lifestyle factors and Sasang constitution types.

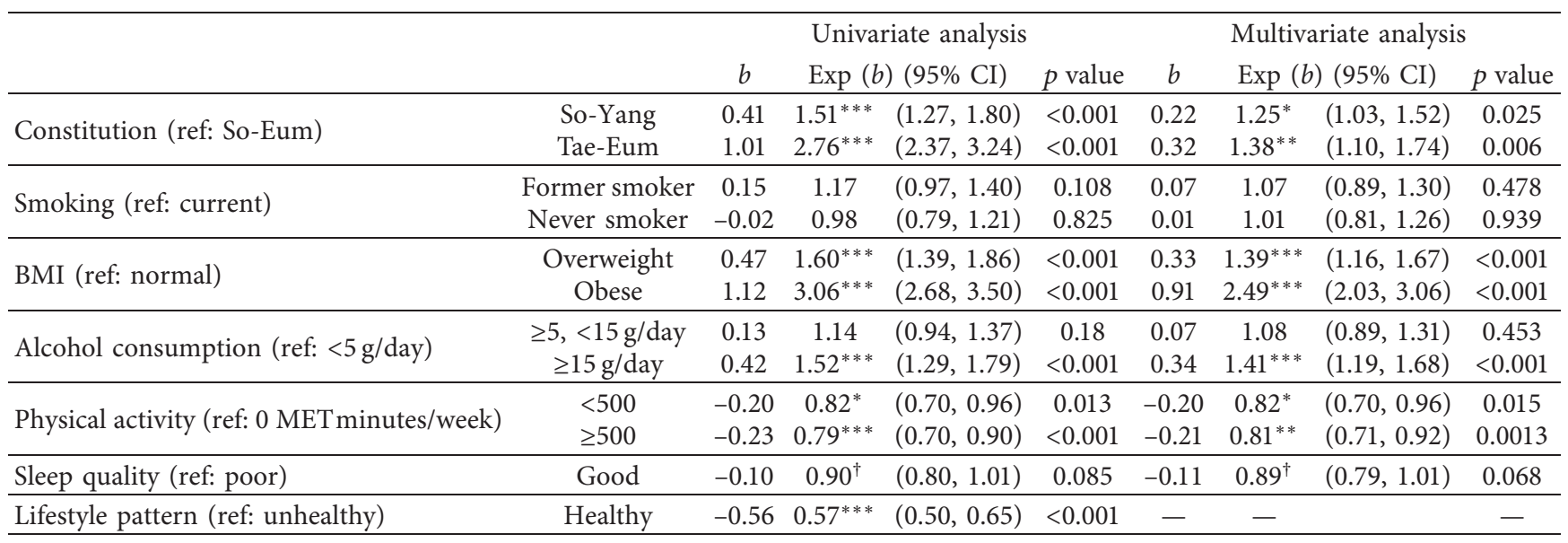

Sex and age were adjusted. ${ }^{\dagger} p<0.1,{ }^{*} p<0.1$, and ${ }^{* * *} p<0.001$. BMI: body mass index, CI: confidence interval, and MET: metabolic equivalents.

In the unhealthy lifestyle group, SY (OR 2.21, 95\% CI: $1.50,3.30)$ and TE (OR 3.64, 95\% CI: 2.55, 5.29) types showed significantly higher odds of hypertension compared with the SE type. In the health lifestyle pattern, higher odds of hypertension were significant in the SY (OR 1.34, 95\% CI: $1.09,1.64$ ) and TE (OR 2.35, 95\% CI: 1.96,
2.83) types compared with the SE type. The difference in ORs among Sasang constitution types was decreased with a healthy lifestyle (Table 4(B)).

Compared with the SE type and the unhealthy lifestyle group, the risk of hypertension was significantly higher in the groups with the SY type and unhealthy lifestyle (OR 2.17, 
TABLE 3: Odds ratios of hypertension for lifestyle factors in each Sasang constitution type.

\begin{tabular}{lccccccc}
\hline & & & \multicolumn{2}{c}{ So-Eum } & \multicolumn{2}{c}{ So-Yang } & \multicolumn{2}{c}{ Tae-Eum } \\
\hline \multirow{2}{*}{ Smoking (ref: current) } & Past smoker & 0.62 & $(0.36,1.07)$ & 1.01 & $(0.70,1.45)$ & 1.21 & $(0.94,1.55)$ \\
& Never smoker & 0.96 & $(0.53,1.71)$ & 0.82 & $(0.52,1.29)$ & 1.11 & $(0.83,1.48)$ \\
\hline \multirow{2}{*}{ BMI (ref: normal) } & Overweight & $1.67^{\dagger}$ & $(0.97,2.86)$ & $1.38^{* *}$ & $(1.09,1.74)$ & $1.47^{*}$ & $(1.03,2.11)$ \\
& Obese & - & - & $1.53^{*}$ & $(1.00,2.33)$ & $2.85^{* * *}$ & $(2.02,4.04)$ \\
\hline \multirow{2}{*}{ Alcohol consumption (ref: $<5$ g/day) } & $\geq 5,<15$ g/day & 1.12 & $(0.62,1.99)$ & 0.88 & $(0.59,1.31)$ & 1.15 & $(0.90,1.47)$ \\
& $\geq 15$ g/day & 1.06 & $(0.64,1.75)$ & $1.37^{\dagger}$ & $(0.97,1.93)$ & $1.47^{* * *}$ & $(1.18,1.84)$ \\
\hline \multirow{2}{*}{ Physical activity (ref: 0 METminutes/week) } & $<500$ & 0.91 & $(0.61,1.35)$ & 0.94 & $(0.67,1.31)$ & $0.75^{* *}$ & $(0.61,0.92)$ \\
& $\geq 500$ & 0.83 & $(0.60,1.15)$ & 0.85 & $(0.66,1.11)$ & $0.78^{* *}$ & $(0.66,0.93)$ \\
\hline \multirow{2}{*}{ Sleep quality (ref: poor) } & Good & 1.11 & $(0.82,1.50)$ & 0.83 & $(0.65,1.05)$ & $0.86^{\dagger}$ & $(0.73,1.01)$ \\
\hline
\end{tabular}

Sex and age were adjusted. ${ }^{\dagger} p<0.1,{ }^{*} p<0.05,{ }^{* *} p<0.01$, and ${ }^{* * *} p<0.001$.

TABLE 4: Odds ratios for hypertension by lifestyle factors for each Sasang constitution type (A), by Sasang constitution type with each lifestyle pattern (B), and by Sasang constitution types and lifestyle factors (C).

\begin{tabular}{|c|c|c|c|}
\hline & So-Eum & So-Yang & Tae-Eum \\
\hline \multicolumn{4}{|c|}{ (A) Lifestyle factors for each Sasang constitution type } \\
\hline Unhealthy lifestyle & 1.00 & 1.00 & 1.00 \\
\hline Healthy lifestyle & $0.94(0.60,1.48)$ & $0.62^{* * *}(0.48,0.81)$ & $0.69^{* * *}(0.58,0.81)$ \\
\hline \multicolumn{4}{|c|}{ (B) Sasang constitution type with each lifestyle pattern } \\
\hline Unhealthy lifestyle & 1.00 & $2.21^{* * *}(1.50,3.30)$ & $3.64^{* * *}(2.55,5.29)$ \\
\hline Healthy lifestyle & 1.00 & $1.34^{* *}(1.09,1.64)$ & $2.35^{* * *}(1.96,2.83)$ \\
\hline \multicolumn{4}{|c|}{ (C) Sasang constitution types and lifestyle factors } \\
\hline Unhealthy lifestyle & 1.00 & $2.17^{* * *}(1.47,3.26)$ & $3.70^{* * *}(2.58,5.39)$ \\
\hline Healthy lifestyle & $1.06(0.72,1.58)$ & $1.39^{\dagger}(0.96,2.06)$ & $2.45^{* * *}(1.70,3.58)$ \\
\hline
\end{tabular}

Sex and age were adjusted. ${ }^{* * *} p<0.001,{ }^{* *} p<0.01$, and ${ }^{\dagger} p<0.1$.

95\% CI: 1.47, 3.26), TE type and unhealthy lifestyle (OR 3.70, 95\% CI: 2.58, 5.39), and TE type with healthy lifestyle (OR $2.45,95 \% \mathrm{CI}: 1.70,3.58)$. The higher risk was marginally significant in the SY type with healthy lifestyle (OR 1.39, 95\% CI: $0.96,2.06$ ) group (Table $4(\mathrm{C})$ ).

For sensitivity analysis, the participants were divided into three lifestyle groups including unhealthy ( $0-1$ point), average ( $2-3$ points), and healthy lifestyle ( $4-5$ points) and compared. Average (OR 0.64, 95\% CI: 0.54, 0.77) and healthy lifestyle (OR 0.42, 95\% CI: 0.34, 0.51) showed significantly lower risk for hypertension than unhealthy lifestyle (Table S1A). The difference among Sasang constitutional types was also decreased in health lifestyle since the risk in TE types was 3.13 in average lifestyle compared with the SE type, but it was only 2.07 in healthy lifestyle (Table S1B).

\section{Discussion}

Sasang constitution types are associated with different prevalence of various diseases, from functional dyspepsia [35] to metabolic syndrome $[13,17]$. Even though the TE type has the lowest cancer incidence compared with other types [20], TE and SY types are usually reported to have a higher risk for many diseases than the SE type. In studies of metabolic syndrome, SY and TE types have showed higher prevalence than the SE type $[13,17]$. For diabetes, TE and SY types have higher risk than the SE type, with only significantly higher risk for the TE type [18]. A recent systematic review reported that the highest prevalence of metabolic syndrome was in the TE type, whereas irritable bowel syndrome was most common in the SE type [36].

For hypertension, the TE type has higher risk than other constitution types, according to the findings of previous studies. One study on the risk of hypertension by constitution reported that individuals of the TE type had significantly higher risk than those of the SE type, even after adjusting for age, sex, BMI, activity, smoking, alcohol consumption, and diabetes [14]. Another study also found that people with the TE type had higher risk of hypertension than non-TE types [15]. TE type is also associated with increased risk of prehypertension [16].

In the book of Dongyisusebowon, each Sasang constitution has specific typical imbalances in the functions of the organ systems [37]. Pham et al. reported that individuals with the TE constitution produce relatively less heat, which indicates that the TE constitution has poorer metabolic efficacy than other constitutional types and has a relatively weak lung function, as the lung function represents catabolism [38]. Shim et al. also reported that the weak lung function of the TE constitution is related to a weak maximal aerobic capability, which causes an increased thermal equilibrium weight. Therefore, individuals with the TE constitution are exposed to cold stress, which leads to weight gain due to a compensatory mechanism [39]. Because obesity is associated with cardiovascular disease due to hypertension and high concentrations of blood lipids and blood glucose [40], this may lead to a high risk of cardiovascular disease in those with the TE constitution. 
The results of this study revealed that the TE type had higher risk of hypertension than SY or SE types. One study reported significantly higher odds of prehypertension in the TE type than the SE type in a crude model, but this was not significant after adjusting for age and BMI [16]. In the present study, the difference in risk among Sasang constitution types, especially for the TE type, was still significant after adjusting for age, sex, and lifestyle factors including BMI. Several studies have reported that personalized medicine, which considers individual characteristics, is important for the management of hypertension [41, 42]. Sasang constitution could be used as an index to predict and properly manage hypertension.

We also found that the difference in risk of hypertension among Sasang constitution types decreased with a healthy lifestyle. The OR for the TE type with an unhealthy lifestyle was 3.70, compared with the SE type and an unhealthy lifestyle; however, in individuals with the TE type who maintained a healthy lifestyle, the risk of hypertension was lower (OR 2.45). Likewise, the higher risk of hypertension in the SY type (compared with the SE type) also declined when individuals with the SY type maintained a healthy life. Engaging in a healthy lifestyle that includes refraining from smoking and alcohol consumption as well as maintaining appropriate weight, physical activity, and sleep might decrease the risk for various diseases caused by inherent characteristics, such as Sasang constitution types.

This study demonstrated that the effect of lifestyle factors on health is different according to Sasang constitution types. Another study investigating Sasang constitution type for risk prediction of metabolic syndrome reported different significant predictors by the constitution type. In that study, smoking and alcohol consumption were significant factors in TE and SY types but not in the SE type [21]. In addition to lifestyle factors, Cha et al. reported that the genetic effects on body mass differ according to Sasang constitution types, especially the TE type [43]. Further studies on the different impacts of genetic and behavioral factors are needed.

This study had several limitations. First, this study was based on a cross-sectional design. Cross-sectional studies have inherent limitations; thus, the order of hypertension and lifestyle factors could not be clarified. The higher risk of former smoker compared with current smoker might be caused by this limitation. However, our findings on the effect of lifestyle factors on hypertension were similar to those of previous studies [27, 44]. Second, diet was not included in the analysis, even though diet is associated with hypertension prevalence [27]. Third, the present analysis was not adjusted for chronic diseases such as renal disease, thyroid disorders, and family history of hypertension, as such information was either not acquired or not appropriate. For example, the collected information about chronic disease included whether a participant had a certain chronic disease but did not specify whether the disease was cured or still being treated. Further prospective studies should include data on diet and chronic disease.
Previous studies have investigated the inheritability and genetics of the Sasang constitutional types. Hur et al. analyzed the overlap of genetic and environmental factors among Sasang constitutions and reported that the pleiotropic mechanism of genes may mediate the phenotypic associations among constitutions [45]. In addition, Kim et al. showed that the biologic pathways and core node genes are associated with Sasang constitutions [46], and another study found a significant relationship between genetic loci and Sasang constitutions [47], whereas one systematic review reported no conclusive genotype factors related to Sasang constitution types [48], another study proposed objective indexes of the Sasang constitution type, such as the obesity-risk variant in intron 1 of the fat mass and the obesity-associated gene [49]. With an objective and reliable index, personalized prevention or treatment methods based on Sasang constitution would be more useful and beneficial.

\section{Conclusion}

In this study, SY and TE types showed significantly higher risk for hypertension compared with the SE type even after adjustment of lifestyle factors. Individuals with healthy lifestyles had significantly lower risk of hypertension compared to those with unhealthy lifestyles in SY and TE types but not in the SE type. The difference in hypertension risk among the constitution types decreased with a healthy lifestyle.

\section{Abbreviations}

BMI: Body mass index

CI: Confidence interval

MET: Metabolic equivalents

OR: Odds ratio.

\section{Data Availability}

The datasets used and/or analyzed during the current study are available from Korean Medicine Data Center (https:// kdc.kiom.re.kr) on reasonable request.

\section{Ethical Approval}

This study was approved by the Korea Institute of Oriental Medicine Research Ethics Committee (I-1809/006/001).

\section{Conflicts of Interest}

All authors declare that they have no conflicts of interest.

\section{Authors' Contributions}

JEP designed the study, conducted data analysis, and wrote the manuscript. SC participated in data collection and analysis. SWL supervised the study. All authors critically revised the manuscript. All authors read and approved the final manuscript. 


\section{Acknowledgments}

This research was supported by Establishment of Korean Medicine Genome and Epidemiology Infrastructure (KSN1713091) and KIOM under Grant KSN1713091 from Korea Institute of Oriental Medicine.

\section{Supplementary Materials}

Table S1: odds ratios for hypertension by three levels of lifestyle factors for each Sasang constitution type (A) and by Sasang constitution type with each lifestyle pattern (B). (Supplementary Materials)

\section{References}

[1] C. Armstrong and Joint national committee, "JNC 8 guidelines for the management of hypertension in adults," American Family Physician, vol. 90, no. 7, pp. 503-504, 2014.

[2] K. T. Mills, J. D. Bundy, T. N. Kelly et al., "Global disparities of hypertension prevalence and control: a systematic analysis of population-based studies from 90 Countries," Circulation, vol. 134, no. 6, pp. 441-450, 2016.

[3] M. H. Forouzanfar, P. Liu, G. A. Roth et al., "Global burden of hypertension and systolic blood pressure of at least 110 to 115 mm Hg, 1990-2015," Journal of the American Medical Association, vol. 317, no. 2, pp. 165-182, 2017.

[4] Statistics Korea, Prevalence of Chronic Disease Secondary Prevalence of Chronic Disease, Statistics Korea, Daejeon, South Korea, 2019.

[5] Korea Centers for Disease Control \& Prevention, Chronic Disease Factbook 2017, Korea Centers for Disease Control \& Prevention, Cheongju, Chungcheongbuk-do, South Korea, 2017.

[6] World Health Organization, A Global Brief on Hypertension, World Health Organization, Geneva, Switzerland, 2019.

[7] Z. Shafieyan, M. Qorbani, B. Rastegari Mehr et al., "Association between lifestyle and hypertension in patients referred to health care centers of ilam city in 2014," Global Journal of Health Science, vol. 8, no. 6, pp. 161-167, 2015.

[8] J. Díaz-Gutiérrez, L. Ruiz-Estigarribia, M. Bes-Rastrollo, M. Ruiz-Canela, J. M. Martin-Moreno, and M. A. MartínezGonzález, "The role of lifestyle behaviour on the risk of hypertension in the SUN cohort: the hypertension preventive score," Preventive Medicine, vol. 123, pp. 171-178, 2019.

[9] S. Iliodromiti, N. Ghouri, C. A. Celis-Morales, N. Sattar, M. A. Lumsden, and J. M. Gill, "Should physical activity recommendations for South asian adults Be ethnicity-specific? Evidence from a cross-sectional study of South asian and white European men and women," PLoS One, vol. 11, no. 8, Article ID e0160024, 2016.

[10] L. Cohen, G. C. Curhan, and J. P. Forman, "Influence of age on the association between lifestyle factors and risk of hypertension," Journal of the American Society of Hypertension, vol. 6, no. 4, pp. 284-290, 2012.

[11] H. Chae, I. K. Lyoo, S. J. Lee et al., "An alternative way to individualized medicine: psychological and physical traits of Sasang typology," The Journal of Alternative and Complementary Medicine, vol. 9, no. 4, pp. 519-528, 2003.

[12] J. Y. Kim and D. D. Pham, "Sasang constitutional medicine as a holistic tailored medicine," Evidence-Based Complementary and Alternative Medicine, vol. 6, no. 1, pp. 11-19, 2009.
[13] K. H. Song, S. G. Yu, and J. Y. Kim, "Prevalence of metabolic syndrome according to sasang constitutional medicine in Korean subjects," Evidence-Based Complement and Alternative Medicine, vol. 2012, Article ID 646794, 8 pages, 2012.

[14] S. K. Lee, D. W. Yoon, S. W. Lee et al., "Association of sasang constitutional types with incident hypertension: a 12-year follow-up study," The Journal Of Alternative And Complementary Medicine, vol. 22, no. 9, pp. 706-712, 2016.

[15] J. Lee, J. Lee, E. Lee, J. Yoo, Y. Kim, and B. Koh, "The Sasang constitutional types can act as a risk factor for hypertension," Clinical and Experimental Hypertension, vol. 33, no. 8, pp. 525-532, 2011.

[16] E. Jang, Y. Baek, Y. Kim, K. Park, and S. Lee, "Sasang constitution may act as a risk factor for prehypertension," BMC Complementary and Alternative Medicine, vol. 15, no. 1, p. 231, 2015.

[17] S. K. Lee, D. W. Yoon, K.-M. Choi et al., "The association of Sasang constitutional types with metabolic syndrome: a pooled analysis of data from three cohorts," European Journal of Integrative Medicine, vol. 8, no. 3, pp. 227-234, 2016.

[18] T.-G. Lee, B. Koh, and S. Lee, "Sasang constitution as a risk factor for diabetes mellitus: a cross-sectional study," EvidenceBased Complementary and Alternative Medicine, vol. 6, no. s1, pp. 99-103, 2009.

[19] E. Jang, Y. Baek, K. Park, and S. Lee, "Could the Sasang constitution itself be a risk factor of abdominal obesity?," BMC Complementary and Alternative Medicine, vol. 13, p. 72, 2013.

[20] J. Lee, W. Kang, J. Cho, C. Cho, H. Yoo, and C. Son, "Cancer incidence varies significantly depending on sasang constitution of traditional Korean medicine," Journal of Traditional Chinese Medicine, vol. 33, no. 3, pp. 312-315, 2013.

[21] S. Lee, S. K. Lee, J. Y. Kim, N. Cho, and C. Shin, "Sasang constitutional types for the risk prediction of metabolic syndrome: a 14-year longitudinal prospective cohort study," BMC Complementary and Alternative Medicine, vol. 17, no. 1, p. 438, 2017.

[22] S. K. Lee, D. W. Yoon, S. W. Lee, J. Y. Kim, J. K. Kim, and C. Shin, "Non-alcoholic fatty liver disease among sasang constitutional types: a population-based study in Korea," BMC Complementary and Alternative Medicine, vol. 15, p. 399, 2015.

[23] Korea Institute of Oriental Medicine, Korean Medicine Data Center, Korea Institute of Oriental Medicine, Daejeon, South Korea, 2019.

[24] National Institute of Health, KoGES_Ansan and Ansung Study, National Institute of Health, Bethesda, MA, USA, 2019.

[25] Y.-H. Baek, E.-S. Jang, K.-H. Park, J.-H. Yoo, H.-J. Jin, and S.-W. Lee, "Development and validation of brief KS-15 (Korea sasang constitutional diagnostic questionnaire) based on body shape, temperament and symptoms," Journal of Sasang Constitutional Medicine, vol. 27, no. 2, pp. 211-221, 2015.

[26] J.-L. Pepin, A.-L. Borel, R. Tamisier, J.-P. Baguet, P. Levy, and Y. Dauvilliers, "Hypertension and sleep: overview of a tight relationship," Sleep Medicine Reviews, vol. 18, no. 6, pp. 509-519, 2014.

[27] J. P. Forman, M. J. Stampfer, and G. C. Curhan, "Diet and lifestyle risk factors associated with incident hypertension in women," Jama, vol. 302, no. 4, pp. 401-411, 2009.

[28] K. M. Diaz, J. N. Booth III, D. A. Calhoun et al., "Healthy lifestyle factors and risk of cardiovascular events and mortality in treatment-resistant hypertension: the reasons for geographic and racial differences in stroke study," Hypertension, vol. 64, no. 3, pp. 465-471, 2014. 
[29] World Health Organization, The Asia-Pacific Perspective: Redefining Obesity and its Treatment. 2000, World Health Organization, Geneva, Switzerland, 2019.

[30] Office of Disease Prevention and Health Promotion, Translating Scientific Evidence about Total Amount and Intensity of Physical Activity into Guidelines, Office of Disease Prevention and Health Promotion, Washington, D.C., USA, 2018.

[31] D. J. Buysse, C. F. Reynolds III, T. H. Monk, S. R. Berman, and D. J. Kupfer, "The pittsburgh sleep quality Index: a new instrument for psychiatric practice and research," Psychiatry Research, vol. 28, no. 2, pp. 193-213, 1989.

[32] M. Sotos-Prieto, A. Baylin, H. Campos, L. Qi, and J. Mattei, "Lifestyle cardiovascular risk score, genetic risk score, and myocardial infarction in hispanic/latino adults living in Costa Rica," Journal of the American Heart Association, vol. 5, no. 12, 2016.

[33] Y. Li, A. Pan, D. D. Wang et al., "Impact of healthy lifestyle factors on life expectancies in the US population," Circulation, vol. 138, no. 4, 2018.

[34] Q.-L. Zhang, L.-G. Zhao, H.-L. Li et al., "The joint effects of major lifestyle factors on colorectal cancer risk among Chinese men: a prospective cohort study," International Journal of Cancer, vol. 142, no. 6, pp. 1093-1101, 2018.

[35] Y. J. Kim, Y. C. Ahn, and C. G. Son, "Sasang constitution affects the prevalence of functional dyspepsia," BMC Complementary and Alternative Medicine, vol. 15, no. 1, p. 150, 2015.

[36] H.-Y. Lee, W.-J. Lee, H.-W. Kim et al., "A systematic review on sasang constitutional type-associated susceptibility to disorders in Korea," The Journal of Alternative and Complementary Medicine, vol. 22, no. 12, pp. 950-956, 2016.

[37] S. Shin, Y.-H. Kim, and M.-W. Hwang, "Diagnosis and treatment principle in Sasang medicine: original symptom," Integrative Medicine Research, vol. 5, no. 2, pp. 99-104, 2016.

[38] D. D. Pham and C. H. Leem, "Body temperature regulation: sasang typology-based perspective," Integrative Medicine Research, vol. 4, no. 4, pp. 189-194, 2015.

[39] E. B. Shim, S. W. Lee, J. Y. Kim, C. H. Leem, and Y. E. Earm, "Taeeum-type people in Sasang constitutional medicine have a reduced mitochondrial metabolism," Integrative Medicine Research, vol. 1, no. 1, pp. 41-45, 2012.

[40] L. Akil and H. A. Ahmad, "Relationships between obesity and cardiovascular diseases in four southern states and Colorado," Journal of Health Care for the Poor and Underserved, vol. 22, no. 4A, pp. 61-72, 2011.

[41] C. Savoia, M. Volpe, G. Grassi, C. Borghi, E. Agabiti Rosei, and R. M. Touyz, "Personalized medicine-a modern approach for the diagnosis and management of hypertension," Clinical Science, vol. 131, no. 22, pp. 2671-2685, 2017.

[42] J. B. Byrd, "Personalized medicine and treatment approaches in hypertension: current perspectives," Integrated Blood Pressure Control, vol. 9, pp. 59-67, 2016.

[43] S. Cha, I. Koo, B. L. Park et al., "Genetic effects of FTO and MC4R polymorphisms on body mass in constitutional types," Evidence-Based Complement and Alternative Medicine, vol. 2011, Article ID 106390, 7 pages, 2011.

[44] Q. Wei, J. Sun, J. Huang et al., "Prevalence of hypertension and associated risk factors in dehui city of jilin province in China," Journal of Human Hypertension, vol. 29, no. 1, pp. 64-68, 2015.

[45] Y.-M. Hur, S. Lee, and H.-J. Jin, "Genetic and environmental overlaps among sasang constitution types: a multivariate twin study," Twin Research and Human Genetics, vol. 21, no. 6, pp. 518-526, 2018.
[46] B.-Y. Kim, S.-G. Yu, J.-Y. Kim, and K. H. Song, "Pathways involved in sasang constitution from genome-wide analysis in a Korean population," The Journal of Alternative and Complementary Medicine, vol. 18, no. 11, pp. 1070-1080, 2012.

[47] H.-H. Won, S. Lee, E. Jang et al., "A genome-wide scan for the Sasang constitution in a Korean family suggests significant linkage at chromosomes 8q11.22-23 and 11q22.1-3," The Journal of Alternative and Complementary Medicine, vol. 15, no. 7, pp. 765-769, 2009.

[48] K. Sohn, A. Jeong, M. Yoon, S. Lee, S. Hwang, and H. Chae, "Genetic characteristics of Sasang typology: a systematic review," Journal of Acupuncture and Meridian Studies, vol. 5, no. 6, pp. 271-289, 2012.

[49] S. Cha, H. Yu, A. Y. Park, S. A. Oh, and J. Y. Kim, "The obesity-risk variant of FTO is inversely related with the SoEum constitutional type: genome-wide association and replication analyses," BMC Complementary and Alternative Medicine, vol. 15, p. 120, 2015. 